\title{
Efecto del alimento sobre variables productivas de dos especies de Cladóceros bajo condiciones de laboratorio
}

\section{Effect of food intake on productive variables of two species of cladocera, under laboratory conditions}

\author{
Angélica Otero P, M.Sc, Marcela Muñoz P, Biol, Víctor Medina-Robles,* M.Sc, \\ Pablo Cruz-Casallas, Ph.D.
}

\begin{abstract}
Universidad de los Llanos, Instituto de Acuicultura. Grupo de Investigación sobre Reproducción y Toxicología de Organismos Acuáticos - GRITOX, km 12 vía Puerto López, Villavicencio, Colombia. *Correspondencia: mauriciomedina77@gmail.com
\end{abstract}

Recibido: Julio de 2012; Aceptado: Abril de 2013.

\section{RESUMEN}

Objetivo. Evaluar los efectos de diferentes tipos de alimentos sobre la productividad de Diaphanosoma sp., y Alona sp bajo condiciones de laboratorio. Materiales y métodos. Juveniles de Diaphanosoma sp., y Alona sp., fueron sembrados en acuarios de $20 \mathrm{~L}$ a una densidad de 6 org L $^{-1}$ y mantenidos con aireación constante y fotoperíodo 12:12 (luz : oscuridad). Se alimentaron con Saccharomyces cereviseae y Chlorella vulgaris a concentración de $4 \times 10^{5} \mathrm{Cel} / \mathrm{ml}$ cada uno, en tres combinaciones: 1 . C. vulgaris, 2. S. cereviseae, 3. C. vulgaris + S. cereviseae en proporciones iguales; de cada tratamiento se realizaron 3 réplicas. Con el propósito de determinar la densidad poblacional, cada 3 días y durante un período de 27 días, y se midió la concentración de individuos por ml. Resultados. Se obtuvieron mejores resultados en el tratamientos de microalgas + levadura, con valores de TCE $=0.1323 \pm 0.07 \mathrm{y}$ $0.1598 \pm 0.04$ días $^{-1} ; R=0.010 \pm 0.004$ y $0.019 \pm 0.01$ org ml $^{-1}$ día $^{-1}, \mathrm{TD}=5.2 \pm 0.5$ y $4.3 \pm 0.38$ días para Diaphanosoma sp., y Alona sp., respectivamente, pero sin presentar diferencias significativas $(p>0.05)$. Conclusiones. La dieta mixta de levadura y microalgas produjo mayor eficiencia productiva en las dos especies estudiadas, constituyendo una alternativa de fuente de alimento vivo para la acuicultura.

Palabras clave: Chlorella vulgaris, cladóceros, zooplancton (Fuente: AIMS).

\section{ABSTRACT}

Objective. Assess the effects of different types of food intake on the productivity of Diaphanosoma sp. and Alona sp. under laboratory conditions. Materials and methods. Young Diaphanosoma sp. and Alona sp. were planted in $20 \mathrm{~L}$ aquariums at a density of $6 \mathrm{org} \mathrm{L}^{-1}$ and maintained with constant aeration and a photoperiod 12:12 (light: dark). The organisms were fed with Saccharomyces cereviseae and Chlorella vulgaris at a concentration of $4 \times 10^{5}$ cells $/ \mathrm{ml}$ in three combinations: 1 . C. vulgaris, 2. S. cereviseae 3. C. vulgaris + S. cereviseae); 3 replicas were carried out for each treatment. To determine the population density, every 3 days over a 27 day period, the concentration of 
individuals per $\mathrm{ml}$ was measured using a Bogorov camera. Results. The best results were obtained in treatments with microalgae and yeast, with values of TCE $=0.1323 \pm 0.07$ and $0.1598 \pm 0.04$ days $^{-1}$, $\mathrm{R}=0.010 \pm 0.004$ and $0.019 \pm 0.01$ org $\mathrm{ml}-1$ days $-1, \mathrm{TD}=5.2 \pm 0.5$ and $4.3 \pm 0.38$ days for Diaphanosoma sp., and Alona sp., respectively, but without significant differences $(p>0.05)$. Conclusions. The mixed diet of yeast and microalgae produced greater productive efficiency in the studied species, constituting an alternative source of live feed for aquaculture.

Key words: Chlorella vulgaris, cladocerans, zooplankton (Source: AIMS).

\section{INTRODUCCIÓN}

El zooplancton vivo constituye una fuente importante de alimento para las larvas de crustáceos y peces, debido a su tamaño reducido y valor nutricional; siendo muchas veces un factor limitante en los procesos de larvicultura y alevinaje de las especies cultivadas. Entre los organismos zooplanctónicos, los cladóceros se destacan no sólo por su reproducción partenogenética y ciclo corto de vida, sino también por su capacidad de producción en períodos cortos de tiempo $(1,2)$; además, por ser una fuente importante de aminoácidos, lípidos, ácidos grasos esenciales, vitaminas y enzimas (peptidasas, proteinasas, lipasas y amilasas) que sirven como exoenzimas en el intestino de las larvas de los peces (3). De igual manera presentan otras ventajas en larvicultura, no afectan la calidad del agua y sus características se mantienen por largos períodos. Dentro de los cladóceros se destacan los géneros Daphnia y Moina, los cuales son de gran importancia en la piscicultura (3).

El cultivo de cladóceros ofrece la ventaja de generar un gran número de individuos en cortos períodos de tiempo, siempre y cuando se le proporcione al cultivo las condiciones adecuadas de temperatura, calidad de agua y alimentación (4). Según algunos autores $(5,6)$, el valor nutricional y la cantidad de alimento suministrada, influye sobre la tasa de reproducción y sobre la frecuencia reproductiva de cladóceros y rotíferos; determinando el crecimiento de la población, por lo que se ha observado, en muchas especies de zooplancton, una relación directa entre la producción de huevos partenogenéticos y la cantidad de alimento disponible (7).

Existen varios métodos para evaluar los efectos del tipo de alimento sobre el crecimiento de las poblaciones cultivadas de zooplancton, entre los que se encuentran el análisis bromatológico y la cuantificación de la densidad de la población (8); sin embargo, el uso de especies nativas de cladóceros en la alimentación de larvas es aún muy limitado, debido a la ausencia de protocolos de cultivo que permitan su producción a escala comercial, con la calidad y en la cantidad necesaria para suplir los requerimientos básicos de las diferentes especies de peces (3). Por lo anterior, el objetivo del presente trabajo fue evaluar diferentes tipos de alimento sobre algunas variables productivas de dos especies de cladóceros (Diaphanosoma sp., y Alona sp.), bajo condiciones experimentales de de laboratorio.

\section{MATERIALES Y MÉTODOS}

Localización y descripción del área de estudio. La investigación se llevó a cabo en el Laboratorio de Producción de Alimento Vivo del Instituto de Acuicultura de la Universidad de los Llanos, localizado a $4 \mathrm{~km}$ de la ciudad de Villavicencio. La zona de estudio presenta una altura promedio de 418 m.s.n.m, temperatura de $25^{\circ} \mathrm{C}$, precipitación pluvial anual de $4050 \mathrm{~mm}$ y humedad relativa del $75 \%$.

Cultivo de microalgas. La cepa de microalga Chlorella vulgaris fue obtenida del laboratorio de alimento vivo del Instituto de Acuicultura de la Universidad de los Llanos. Para el cultivo se utilizó como medio un fertilizante inorgánico del complejo NPK (REMITAL ${ }^{\circledR} \mathrm{m}-17-6-18$ ) a razón de $1 \mathrm{~g} \mathrm{~L}^{-1}(9)$. El cultivo se mantuvo bajo condiciones controladas de temperatura de $18 \pm 1^{\circ} \mathrm{C}$, 24 horas luz y aireación constante.

Material biológico. Se utilizaron individuos juveniles de las especies Diaphanosoma sp., y Alona sp. proporcionados por el Laboratorio de Alimento Vivo de la Universidad de los Llanos. Para evaluar los efectos del alimento sobre algunas variables productivas se seleccionaron aleatoriamente juveniles de cladóceros, los cuales fueron ubicados en acuarios de vidrio con capacidad de $20 \mathrm{~L}$ a una densidad inicial de 6 organismos $\mathrm{L}^{-1}$, conformando 6 tratamientos como se muestra en la tabla 1. Durante el experimento se mantuvo la aireación constante y se manejó un fotoperíodo de 12:12 (luz: oscuridad). Se alimentó una vez al día (10:00 a.m), la densidad del alimento se determinó 
mediante recuento en cámara de Neubauer (Brightline "Boeco ${ }^{\circledR ", ~ G e r m a n y), ~ u t i l i z a n d o ~ u n ~}$ microscopio óptico ("Nikon ${ }^{\circledR "}$ Eclipse E200, Japón) con aumento de 40x y se utilizó la fórmula propuesta por Sipaúba -Tavares et al (7):

Número de células contadas

$$
10 \times 4 \times 10^{-6}
$$

Tabla 1. Tratamientos evaluados durante el cultivo de Alona sp. y Diaphanosoma sp., bajo condiciones de laboratorio.

\begin{tabular}{ccc}
\hline $\begin{array}{c}\text { Especie de } \\
\text { Cladócero }\end{array}$ & Tratamientos & $\begin{array}{c}\text { Alimento } \\
\left(\mathbf{4} \times \mathbf{1 0}^{\mathbf{5}} \mathbf{~ C e l} / \mathbf{m l}\right)\end{array}$ \\
\hline Alona sp. & T1 & C. vulgaris \\
& T2 & S. cerevisiae \\
Diaphanosoma sp. & T3 & C. vulgaris + S. cerevisiae (1:1) \\
& T4 & C. vulgaris \\
& T5 & S. cerevisiae \\
& T6 & C. vulgaris + S. cerevisiae $(1: 1)$ \\
\hline
\end{tabular}

Se emplearon tres réplicas por tratamiento, asignadas aleatoriamente. Durante 27 días, cada 3 días, se midió la densidad del cultivo por medio del recuento en cámara Bogorov para zooplancton.

Se midieron parámetros físicos y químicos del agua, tales como temperatura y $\mathrm{pH}$, por medio de un $\mathrm{pH}$ metro digital (Hanna pHep 5, Mauritius), oxígeno disuelto y conductividad, haciendo uso de una sonda multiparamétrica YSI (YSI 556 Professional Plus Ohaus, USA).

Variables observadas. Como variables de respuesta se evaluaron: Tasa instantánea de crecimiento (TCE), Tiempo de duplicación (TD), Rendimiento $(R)$ y Densidad máxima (Dm), siguiendo las criterios reportados por Prieto et al (3) y Heugens (10):

Tasa instantánea de crecimiento (TCE). Determina el aumento de la población en un período específico.

$\mathrm{TCE}=\frac{\operatorname{LnNt1}-\operatorname{Ln} \mathrm{Nt} 0}{\mathrm{~T}}$

Donde:

Nt1: Número final de cladóceros en ml NtO: Número inicial de cladóceros en $\mathrm{ml}$ T: $\quad$ Tiempo del cultivo

Tiempo de duplicación. Tiempo que tarda la población en duplicarse.
$\mathrm{TD}=\frac{\mathrm{Ln} 2}{\mathrm{~K}}$

Donde:

Ln2: Logaritmo natural de 2

K: Tasa instantánea de crecimiento (TCE).

Rendimiento. Número de organismos por día

$\mathrm{R}=\frac{\mathrm{Nt} 1-\mathrm{Nt0}}{\mathrm{t}}$

Donde:

Nt1: Número final de organismos por $\mathrm{ml}$ Nt0: Número inicial de organismos por $\mathrm{ml}$ t: $\quad$ Tiempo en días.

Densidad máxima (Dm). Densidad máxima de organismos obtenidos en el cultivo Org $\mathrm{L}^{-1}$.

Día de máxima densidad (Dmd). Día de mayor número de organismos en el cultivo.

Densidad final del cultivo. Organismos/ml.

Análisis estadístico. Se realizó la estadística descriptiva expresando los datos como mediaterror estándar de la media (SEM). Para determinar los efectos de los tratamientos sobre las variables físicas y químicas, los resultados fueron sometidos a un análisis de varianza de una vía (ANOVA), previa verificación de los supuestos de homogeneidad de varianza (Prueba de Bartlett y Levene) y normalidad de los datos (Prueba de Kolmogorov- Smirnov). Una vez comprobada la normalidad de los datos fue aplicada una prueba de comparación de Tukey, con un nivel de significancia de $p<0.05$. Los datos fueron analizados con el software SAS versión 8.02 para Windows (1999-2001 por SAS Institute Inc, Cary, NC. USA)

\section{RESULTADOS}

La tabla 2 muestra los resultados del monitoreo de las condiciones físicas y químicas del agua de cultivo. La temperatura no varió significativamente ( $p>0.05)$ entre tratamientos, siendo registrado el menor promedio en el tratamiento T3 y el mayor en el T4 (26.4 \pm 0.2 y $27 \pm 0.1^{\circ} \mathrm{C}$, respectivamente). Con relación al $\mathrm{pH}$, los tratamientos $\mathrm{T} 1$ y $\mathrm{T} 4$ registraron valores inferiores $(p<0.05)$ a los demás tratamientos. Las demás variables no variaron significativamente ( $p>0.05)$ entre tratamientos.

En la figura 1 se observa que ambas especies de cladóceros durante los primeros días del ensayo presentaron una fase de adaptación, 
Otero - Efecto del alimento sobre variables productivas en dos especies de Cladóceros 3645

Tabla 2. Condiciones físicas y químicas del agua utilizada en el cultivo experimental de los cladóceros Alona sp., y Diaphanosoma sp. Los valores corresponden a la media \pm SEM. $n=27$

\begin{tabular}{lcccccc}
\hline Parámetros & T1 & T2 & T3 & T4 & T5 & T6 \\
\hline $\mathrm{T}\left({ }^{\circ} \mathrm{C}\right)$ & $26.7 \pm$ & $26.52 \pm$ & $26.3 \pm$ & $27 \pm$ & $26.79 \pm$ & $27.0 \pm$ \\
& 0.28 & 0.24 & 0.22 & 0.14 & 0.50 & 0.10 \\
$\mathrm{pH}$ & & & & & & \\
& $5.1 \pm$ & $7.64 \pm$ & $7.0 \pm$ & $5.3 \pm$ & $7.6 \pm$ & $7.4 \pm$ \\
$\mathrm{OD}(\mathrm{mg} / \mathrm{L})$ & $2.7 \pm$ & $2.84 \pm$ & $3.0 \pm$ & $3.0 \pm$ & $3.16 \pm$ & $3.1 \pm$ \\
& 0.30 & 0.31 & 0.33 & 0.27 & 0.29 & 0.29 \\
& & & & & & \\
$\mathrm{C}(\mu \mathrm{S} / \mathrm{cm})$ & $224 \pm$ & $184.1 \pm$ & $208 \pm$ & $204 \pm$ & $189 \pm$ & $250 \pm$ \\
& 26.17 & 18.2 & 28.16 & 22.33 & 17.79 & 37.47
\end{tabular}

T: Temperatura, OD: Oxígeno disuelto y C: Conductividad

durante la cual la tasa de crecimiento fue muy baja. A partir del día 15 se observó una tasa de crecimiento moderada, alcanzando una densidad de $115 \pm 110$ y $85 \pm 30$ orgL $^{-1}$ para Diaphanosoma sp., y Alona sp., respectivamente, sin observarse diferencias significativas ( $p>0.05)$. El cultivo de Diaphanosoma sp., alcanzó la máxima densidad ( $550 \pm 500$ orgL $^{-1}$ ) el día 24, mientras que Alona sp., la alcanzó el día 27 con una densidad de $383 \pm 200$ orgL $^{-1}$ presentando diferencias significativas $(p<0.05)$. A partir del día 27 el cultivo de Diaphanosoma sp., registró un descenso en la densidad, mientras que Alona sp., continuó su crecimiento.

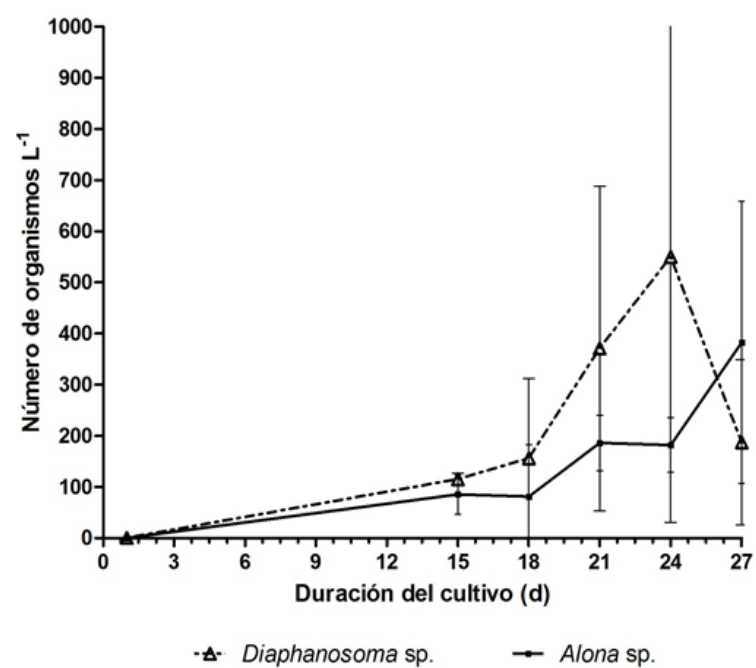

Figura 1. Variación de la densidad poblacional durante 27 d de cultivos de Alona sp., y Diaphanosoma sp., realizados en acuarios de vidrio de $20 \mathrm{~L}$ y alimentadas con una mezcla de microalgas y levaduras. Valores mostrados como media \pm SEM. $n=3$. Los datos de los demás tratamientos no se muestran debido al no crecimiento poblacional.
Para Alona sp., la tasa especifica de crecimiento (TEC), rendimiento $(R)$ y el tiempo de duplicación (TD) fueron de $0.1598 \pm 0.04$ días $^{-1} ; 0.019 \pm 0.01$ org $\mathrm{ml}^{-1}$ día $^{-1}$ y $4.3 \pm 0.38$ días, respectivamente; mientras que para Diaphanosoma sp., fue $0.1323 \pm 0.07$ días $^{-1} ; 0.010 \pm 0.004$ org mL $^{-1}$ día $^{-1}$ y $5.2 \pm 0.5$ días, respectivamente (Tabla 3 ).

Tabla 3. Variables productivas de Diaphanosoma sp., y Alona sp., cultivadas durante 27 dias en acuarios de vidrio y alimentadas con una mezcla de C. vulgaris y $S$. cerevisiae. Los valores corresponden a la media \pm SEM. $n=3$

\begin{tabular}{|c|c|c|}
\hline Variables & $\begin{array}{c}\text { Diaphanosoma sp. } \\
\text { C. vulgaris + } \\
\text { S. cerevisiae }\end{array}$ & $\begin{array}{c}\text { Alona sp. } \\
\text { C. vulgaris + } \\
\text { S. cerevisiae }\end{array}$ \\
\hline TCE $\left(\right.$ días $\left.^{-1}\right)$ & $0.1323 \pm 0.07$ & $0.1598 \pm 0.04$ \\
\hline $\mathrm{R}($ Org ml-1 día-1) & $0.010 \pm 0.004$ & $0.019 \pm 0.01$ \\
\hline TD (días) & $5.2 \pm 0.5$ & $4.3 \pm 0.38$ \\
\hline Dmd (días) & 24 & 27 \\
\hline Dm (Org L-1) & $550 \pm 500$ & $383 \pm 200$ \\
\hline
\end{tabular}

TCE: Tasa Instantánea de Crecimiento; R: Rendimiento; Dm: Densidad Máxima; Dmd: Día de máxima densidad; TD: Tiempo de Duplicación. Los datos de los demás tratamientos no se muestran debido al no crecimiento poblacional.

\section{DISCUSIóN}

La temperatura es un factor importante para el desarrollo y metabolismo de los organismos zooplanctónicos (11). La temperatura promedio en este estudio fue de $26.9^{\circ} \mathrm{C}$, valor que se encuentra dentro del rango de $20-28^{\circ} \mathrm{C}$, reportado como adecuado para el cultivo de cladóceros (7). Sipaúba-Tavares y Bachion (12) reportaron para las especies Diaphanosoma birgei y Moina micrura la utilización de temperaturas de cultivo de alrededor de $25^{\circ} \mathrm{C}$, suministrando alimento a base de microalgas y suplemento de vitaminas; de igual manera para Moina sp., han sido reportadas temperaturas de cultivo entre 25 y $27^{\circ} \mathrm{C}$ y alimentos a base de Scenedesmus sp. (13). Estos autores concluyeron que la temperatura no altera de manera significativa las variables productivas, lo cual coincide con lo observado en este estudio. Las concentraciones de OD del agua no fueron limitantes para el cultivo de cladóceros, esta variable es poco reportada; sin embargo, algunos autores como Rottmann et al (14), reportaron que estos organismos toleran ambientes deficientes o sobresaturados de oxígeno, gracias a su capacidad de sintetizar hemoglobina cuando se someten a ambientes bajos de oxígeno.

$\mathrm{El} \mathrm{pH}$ en los tratamientos T1 y T4 presentó un valor promedio de $5.2 \pm 0.22$; valor que se encuentra por debajo de lo reportado por otros autores. Prieto et al (3) y Romero (13), 
reportaron para Moina sp., $\mathrm{pH}$ de cultivos de 7.62 y 7.00 alimentados con Ankistrodesmus sp., más S. cereviseae y Scenedesmus sp., respectivamente. Esta disminución del $\mathrm{pH}$ puede relacionarse con el incremento en la densidad de la microalga; según Sipaúba-Tavares et al (7), al aumentar la densidad de algas en un medio, el $\mathrm{pH}$ tiende a disminuir. Esta pudo ser una de las razones que incidió para que no se presentara crecimiento en estos tratamientos.

Los cladóceros alimentados con la dieta a base de sólo levaduras, no presentaron crecimiento poblacional, lo cual coincide con los resultados reportados por Peña-Aguado et al (6) en $M$. macrocopa y Ceriodaphnia dubia. Lo anterior puede atribuirse a que estos organismos requieren en su dieta la presencia de ácidos grasos esenciales, tales como: ácidos grasos insaturados (HUFA) y polinsaturados (PUFA) y las levaduras se caracterizan por tener bajos contenidos de PUFA $(20: 5 n-3$ y $22: 6 n-3)(15)$. Autores como Prieto et al (3), recomiendan para cladóceros la utilización de dietas mixtas (microalgas y levadura) o utilizar otros suplementos alimenticios para mantener la estabilidad del cultivo, disminuir los efectos de los productos de degradación de la levadura y suplir la carencia de ciertas vitaminas. Lo anterior, coincide con lo observado en este estudio, siendo más eficientes los cultivos en aquellos tratamientos que incluyeron la mezcla de microalgas y levaduras. De igual manera, $S$. cereviseae ha sido ampliamente estudiada como dieta en otras especies de microcrustáceos: rotíferos, Daphnia sp., Artemia y copépodos, debido a su contenido nutricional (16-18).

Crecimiento poblacional se observó únicamente en los tratamientos en los cuales se utilizó como fuente de alimentos la mezcla de microalgas y levaduras, para ambas especies Diaphanosoma y Alona. Estos resultados coinciden con lo reportado por Prieto et al (3) en Moina sp., alimentadas con Ankistrodesmus sp. y $S$. cereviseae, en proporción $1: 1$, quienes observaron una densidad máxima de $12.300 \pm 300$ org $L^{-1}$. Por su parte, Sipaúba-Tavares et al (11) observaron densidades de 3.15 y 4.593 org L $^{-1}$ con $M$. micrura y D. birgei, respectivamente, alimentados con $A$. gracillis más vitaminas; lo cual confirma, que las dietas mixtas proporcionan mejores resultados y que las levaduras son eficaces cuando se complementan con algas, reduciendo los costos de producción de zooplancton a gran escala (6). De igual manera se han reportado resultados importantes en el cultivo de cladóceros cuando son alimentados con microalgas. Un cultivo de D. birgei alcanzó una densidad de $140 \times 10^{2}$ organismos $\mathrm{L}^{-1}$ alimentados con $A$. gracillis (19), mientras otro de Moina sp., alimentada con Scenedesmus, densidades de 3.020 org L $^{-1}$ (12).

En este estudio, los valores de las variables productivas TCE, TD y $\mathrm{R}$ para ambas especies fueron menores a aquellos reportados por Prieto et al (3) y Romero (12), quienes evaluaron dietas con Ankistrodesmus sp., más Saccharomyces cereviseae y Scenedesmus sp., en Moina sp., encontrando valores de $\mathrm{TCE}=0.36$ y 0.13 ; $\mathrm{TD}=1.94$ y 2.72 y $\mathrm{R}=1.1$ y 0.36 , respectivamente. Estos valores bajos podrían atribuirse al hecho de haber utilizado una densidad poblacional inicial muy baja (6 org $\mathrm{L}^{-1}$ ) para ambas especies, ya que la mayoría de trabajos consultados reportan densidades de siembra de entre 2.000 y 5.000 org $\mathrm{L}^{-1}$.

En conclusión bajo las condiciones experimentales utilizadas, los cladóceros Alona sp., y Diaphanosoma sp., alimentados con la mezcla de microalgas y levadura presentaron el mejor comportamiento productivo. La especie que presentó los mejores resultados con respecto a las variables evaluadas fue Diaphanosoma sp., la cual se recomienda como una especie con gran potencial para el cultivo comercial.

\section{Agradecimientos}

El presente trabajo fue realizado en el marco del convenio $N^{\circ} 106$ de 2010, SENA-Universidad de los Llanos, como parte del proyecto "Evaluación de aspectos reproductivos de zooplancton nativo bajo diferentes fotoperíodos como estrategia para su producción a escala comercial".

\section{REFERENCIAS}

1. Sarma SS, Nandini S. Review of recent ecotoxicological studies on cladocerans. J Environ Sci Health B 2006; 41:1417-1430.
2. Hardy E, Castro JGD. Qualidade nutricional de três especies de clorofíceas cultivadas em laboratório. Acta Amazônica 2000; 30(1):39-47. 
3. Prieto M, Cruz LDI, Morales,M. Cultivo experimental del cladócero Moina $s p$ alimentado con Ankistrodesmus sp y Saccharomyces cerevisiae. Rev MVZ Córdoba 2006; 11:705-714.

4. Sipaúba-Tavares L, Bachion M. Population growth and development of two species of cladocera, Moina micrura and Diaphanosoma birgei, in laboratory. Braz J Biol 2002; 62(4A):701-711.

5. Sarma SSS, Gulati RD, Nandini S. Factors affecting egg-ratios in planktonic rotifer populations under culture conditions. Hydrobiología 2005; 546:361-373.

6. Peña-Aguado F, Nandinia S, Sarma SSS. Differences in population growth of rotifers and cladocerans raised on algal diets supplemented with yeast. Limnológica 2005; 35:298-303.

7. Sipaúba-Tavares L, Rocha O. Produção de Plâncton (fitoplâncton e Zooplâncton) para alimentação de Organismos Aquáticos. $1^{\text {ra }}$ ed. São Carlos, Brasil: RIMA; 2003.

8. Watanabe T, Tamiya T, Oka A, Hirata C, Kitajima C, Fujita S. Improvement of dietary value of live foods for fish larvae by feeding them on $\omega 3$ highly unsaturated fatty acids and fat-soluble vitamins. Nippon Suisan Gakkai Shi 1983; 50:1015-1022.

9. Muñoz-Peñuela M, Ramírez-Merlano JA, Medina-Robles VM, Cruz-Casallas PE, Velasco-Santamaría YM. Efecto del medio de cultivo sobre el crecimiento y el contenido proteico de Chlorella vulgaris. Rev Col Cienc Pec, 2012; 25 (3):438-449.

10. Heugens $E$, Tokkie L, Kraak M, Hendriks A. Van SN and Admiraal W. Population growth of Daphnia Magna under multiple stress conditions: Joint effects of temperature, food, and cadmium. Environ Toxicol Chem 2006; 25:1399-1407.

11. Rodríguez-Estrada JC, Villaseñor F, MartínezJerónimo. Efecto de la temperatura y tipo de alimento en el cultivo de Moina micrura (Kurz, 1984) (Anomopoda:Moinidae) en condiciones de laboratorio. Hydrobiológica 2003; 13(3):239-246.
12. Sipaúba-Tavares LH, Bachion MA. Population growth and development of two species of cladocera, Moina micrura y Diaphanosoma birgei, in laboratory. Braz J Biol 2002; 62(4A): 701-711.

13. Romero TJ. Desarrollo de Moina $s p$ en condiciones de laboratorio alimentada con microalgas cultivadas en residuales pesqueros. REDVET 2009; 10(4):1-14.

14. Rottmann RW, Graves JS, Watson C, Yanong RE. Culture Techniques of Moina: The ideal Daphnia for feeding freshwater fish fry. IFAS Extensión. Florida, USA: University of Florida; 2003.

15. Ocampo LE, Botero M, Restrepo LF. Evaluación del crecimiento de un cultivo de Daphnia magna alimentado con Saccharomyces cerevisiae y un enriquecimiento con avena soya. Rev Colomb Cienc Pecu 2010; 23:78-85.

16. Jiménez D, Rosas J, Velásquez A, Millán J, Cabrera T. Crecimiento poblacional y algunos aspectos biológicos del cladocero Moina macrocopa (Straus, 1820) (Branchiopoda, Anomopoda), alimentado con tres dietas en tres salinidades diferentes. Ciencia 2003; $11: 22-30$.

17. Fazeli Z, Takami G. The best time and concentration for yeast probiotic enrichment of Artemia urmiana Nauplii. Pakistan J Biol Sci 2006; 9:2159-2161.

18. Hamre K, Srivastava A, Ronnestad I, MangorJensen A, Stoss J. Several micronutrients in the rotifer Brachionus $s p$. may not fulfi I the nutritional requirements of marine fish larvae. Aquacult Nutr 2008; 14:51-60.

19. Sipaúba-Tavares, LH, Pereira AML. Large scale laboratory cultures of Ankistrodesmus gracilis (Reisch) Korsikov (Chlorophyta) and Diaphanosoma biergei Korinek, (Cladocera) 1981. Braz J Biol 2008; 68(4):875-883. 